capacity for ribosome binding of different size fragments prepared from the $5^{\prime}$ end of reovirus mRNA. Fragments up to 30 nucleotides in length, which contained the cap but not the initiation codon, did not form stable complexes with ribosomes whereas every fragment which did contain the AUG was able to bind ribosomes.
However, binding efficiency was reduced by removal of either the cap or of sequences comprising the beginning of the coding region. Their conclusion is that, as with prokaryotic ribosome binding sites, the formation of the initiation complex is primarily dependent on the interaction between the AUG and the initiator tRNA.

\title{
Mechanism of transcription termination
}

\author{
from Andrew Travers
}

The regulation of transcription termination is a crucial aspect of the control of gene expression in bacteria and bacteriophage. The termination of an RNA chain requires first that the transcribing polymerase ceases its movement along the DNA template, then that both the completed RNA molecule and RNA polymerase be released. In vitro the DNA sequences signalling termination differ in their effectiveness. At some sites RNA polymerase can terminate by itself, at others RNA release requires an additional protein factor. However, polymerase can still pause at such $\rho$ dependent sites even in the absence of the factor.

What is the nature of the termination signals? Analysis of several $\rho$-independent terminators has revealed that in every case termination occurs distal to a GC-rich region within a run of uridine residues. In addition all such terminators display a region of dyad symmetry in the DNA just proximal to the termination point. In terms of RNA structure this implies that the molecule has the potential to form a stem-loop configuration close to the $3^{\prime}$ terminus. The stability and size of these structures is variable, termination of those RNA molecules possessing the least stable stems being in general more dependent on $\rho$.

The advent of modern techniques has considerably enhanced the direct sequence analysis of DNA regions with particular regulatory functions. In this issue of Nature, (page 414 and 410), Rosenberg et al. and Schwarz et al. present DNA sequences from phage encompassing the first terminator, $t_{121}$, for RNA molecules initiated at a major early promoter, $P_{R}$, while Küpper

Andrew Travers is at the MRC Laboratory of Molecular Biology. Cambridge. et al. (page 423) describe the entire sequence of the $\mathrm{tRNA}_{1}{ }^{\mathrm{Ty}}$ gene distal to the promoter. Termination at both $t_{R 1}$ and the major tRNA ${ }^{T y}$ terminator is absolutely $\rho$-dependent. The most striking difference between these terminators and the $\rho$-independent terminators so far studied is the absence of a terminal run of uridine residues. However $t_{R l}$ does contain a sequence for a comparatively abbreviated stemloop structure just before the 3 -end of the transcript. The functional significance of this region is made apparent by mutations which alter the effectiveness of the $t_{R 1}$ terminators. The cin and cnc mutations both act in cis to enhance or eliminate respectively $\rho$ dependent termination at this site. The single base change in the cin mutant extends the length and stability for the potential stem-loop structure while, by contrast, those of the cnc mutants reduce its stability. Accordingly, Rosenberg et al. speculate that the stability of this structural feature is an important determinant of termination efficiency although they point out that the cin mutation could exert its effect in another way by generating a sequence believed to be important in promoter function and thus stabilising polymerase bound at $t_{\mathrm{Rl}}$.

By contrast at the main tRNA ${ }^{\text {Tyr }}$ terminator no such stable stem-loop structure is apparent. Instead a cluster of four $\mathrm{G} \cdot \mathrm{C}$ base pairs precedes the termination point by one turn of the DNA double helix, a configuration believed to be sufficient to halt transcription temporarily (Maizels Proc. natn Acad. Sci. U.S.A. 70, 3585 ; 1973). In addition a more extensive GC-rich region follows the termination point. Consequently Küpper et al. suggest that a transcribing polymerase can be halted either by a stem-loop structure in the product
RNA or by a stable helical region in the DNA template. However, it is clear that such stable regions are by themselves probably insufficient for chain release to occur. Within the tRNA ${ }^{\mathrm{Tyr}}$ gene a 178 base pair unit is completely repeated three times. Each of these repeats contains both the proximal and distal GC-rich sequences in their entirety. Nevertheless only two out of the three such regions can apparently serve as terminators in vitro. The region that is inactive differs by a $\mathrm{C} \rightarrow \mathrm{T}$ transition from the other two, a change that occurs in a sequence CAATCAA common to both the tRNA $^{\text {Tyr }}$ and $t_{k 1}$ terminators. Küpper et al. suggest that this sequence may be the recognition site for $\rho$-induced chain release.

In phage $\lambda$ the control of terminator function is believed to have a crucial role in the lytic and lysogenic pathways of development. Thus expression of the genes distal to $t_{R 1}$ is dependent on the function of the $\mathrm{N}$ gene product, a protein whose postulated role is that of an anti-terminator at a majority of termination sites (Roberts Nature 224, 1168; 1969). pN will however only mediate antitermination when transcription originates from either of the two early promoters suggesting that recognition sites for $\mathrm{pN}$ (nut) are situated in reasonable proximity to these promoters. One such site nut $\mathrm{k}$, has been identified by Rosenberg et al., as a region of dyad symmetry immediately preceding the terminator $t_{\mathrm{k}}$. Thus the regulator recognition signal and its site of action are, in this instance, in close juxtaposition.

The sequence analysis of the $\lambda$ genome provides tantalising hints about the mechanism of the choice between the lytic and lysogenic modes of development. The $\operatorname{cin}$ and $c n c$ mutants favour the lysogenic and lytic modes respectively, suggesting that reduction of transcription beyond $t_{R 1}$ allows transcription to proceed in the reverse direction from the promoter $P_{\mathrm{RE}}$ whose transcript acts as a messenger for the production of cl repressor. Rosenberg et al. and Schwarz et al. have also defined the sites of the $c Y$ mutations, whose phenotypic consequence is the reduction of repressor synthesis during the establishment of lysogeny. These cis acting mutations apparently define a regulatory sequence annd again occur in regions of dyad symmetry. However it remains unclear whether the $c Y$ region is the promoter site for $P_{\text {HE }}$, a terminator site for a transcript initiated from $P_{R E}$, or a regulator recognition site. The identity of one section of the cY region with part of the $t_{k 1}$ region may indicate a common function but clearly the precise elucidation of the function of these sequences awaits a rigorous biochemical analysis 